Check for updates

Cite this: Anal. Methods, 2019, 11, 1976

\section{A novel stainless steel needle electrode based on porous gold nanomaterials for the determination of copper in seawater $\dagger$}

\author{
Haitao Han, (D) ${ }^{\text {ab }}$ Ying Li, ${ }^{\mathrm{c}}$ Dawei Pan, (D) *ab ${ }^{*}$ Chenchen Wang, ${ }^{a}$ Fei Pan ${ }^{\text {ab }}$ \\ and Xiaoyan Ding ${ }^{\text {ab }}$
}

\begin{abstract}
This study describes the fabrication of a porous gold (P-Au) nanomaterial-functionalized stainless steel acupuncture needle electrode (ANE) with the help of polydopamine (PDA) nanospheres, which were partially sacrificed for the voltammetric determination of copper in seawater. The PDA nanospheres were covered on sensing part, i.e., the needle tip, by the self-polymerization method and employed as a reductant and adhesion agent for the growth of gold nanomaterials with a porous structure and improved stability. The gold nanomaterials with a porous structure were self-assembled and deposited on the ANE with the reduction of $\mathrm{AuCl}_{4}{ }^{-}$by PDA nanospheres, which were gradually sacrificed during this process. Moreover, there was no need to cover the protective layer additionally on the P-Au nanomaterial surface to prevent their peel off due to the strong adhesion capability of PDA. Owing to the excellent electrochemical properties of the P-Au nanomaterials and adhesion ability of the partially sacrificed PDA nanospheres, the modified ANE displayed remarkably improved performance and stability for the voltammetric determination of copper. Under optimal conditions, the linear range and detection limit of the modified ANE for copper ion determination are $0.7 \mathrm{nM}$ to $1000 \mathrm{nM}$ and $0.24 \mathrm{nM}$, respectively. Furthermore, the modified ANE has been successfully applied for the determination of copper in real seawater samples with satisfactory results.
\end{abstract}

Received 30th January 2019 Accepted 11th March 2019

DOI: $10.1039 /$ c9ay00222g

rsc.li/methods have strong advantages, they are usually inconvenient, expensive, and time-consuming. ${ }^{4}$ Comparatively, it is commonly believed that anodic stripping voltammetry (ASV), one of the electrochemical analytical methods, is very efficient and suitable for the detection of $\mathrm{Cu}$ and other heavy metals owing to their high sensitivity and selectivity, rapid and straightforward analytical procedure, portability, low cost, and excellent performance with saline samples (seawater). ${ }^{11-14}$ ASV has been commonly used for the survey of $\mathrm{Cu}$ content in seawater of estuary and coastal systems. ${ }^{15,16}$ In the past decades, mercury was the most widely adopted electrode material for the electrochemical detection of different targets due to its excellent sensitivity and stability. ${ }^{15,16}$ Considering the toxicity of mercury, the use of mercury electrodes has been prohibited in more and more countries and replaced by chemically-modified electrodes for the elemental analysis of metals. ${ }^{11-13,17-21}$ However, considering the low content of $\mathrm{Cu}$ and strong matrix interference in real seawater, most of the chemically-modified electrodes cannot meet the requirements of $\mathrm{Cu}$ detection in practice. Thus, there is an urgent need to fabricate a novel electrode with high sensitivity, anti-interference ability, and stability for the determination of $\mathrm{Cu}$ in seawater.

Acupuncture has been employed therapeutically for thousands of years in China. ${ }^{22}$ Among the acupuncture needles made of different metals, the stainless steel needles are widely adopted due to their advantages of low-cost price and easy 
operation..$^{23}$ It has been reported that acupuncture needles can be used as novel electrodes with a needle shape for electrochemical sensing applications. ${ }^{22,23}$ There is no polishing process necessary for the acupuncture needle electrode (ANE) before electrochemical experiments, and only a cleaning process needed. The needle-shape of the ANE is like a cone, which has a three-dimensional structure. The sensing surface area that the ANE can provide is $\pi r R$, while the traditional glass carbon electrode provides an area of $\pi r^{2}$ ( $r$ is the semidiameter of electrode cross-section and $R$ is the hypotenuse of the cone). Obviously, the $R$ here is larger than $r$. So, if the $r$ is the same, the ANE can provide a larger sensing area than the glass carbon electrode, and the volume of ANE can be much smaller. In other words, the unique aciform structure of ANE provides a larger specific surface area, which is helpful for the combination of different nanomaterials to construct the electrochemical sensing platform with a much superior performance. ${ }^{23}$

Moreover, gold nanomaterials are the popular functional materials for the chemical modification of different electrodes due to their outstanding physical, chemical, and catalytic properties. ${ }^{24-31}$ However, it was found in our previous study that the surface of acupuncture needle was very smooth and that gold nanomaterials were not easily deposited on the needle tip surface. ${ }^{32}$ Therefore, the surface modification of the acupuncture needle tip is essential to achieve the easy fabrication of a nanomaterial-functionalized ANE. Fortunately, polydopamine (PDA) performs well as an adhesion agent to modify the substrate surface properties and improve coating adhesion, and it can be easily deposited on the surface of different electrode materials. ${ }^{33,34}$ The deposited PDA film can contribute as adhesion agent to form a versatile platform for the secondary modification of different functional materials like gold nanomaterials. ${ }^{35,36}$ Due to the reducibility of PDA, the gold nanomaterials can be self-assembled and deposited on the ANE surface in a porous form with partially sacrificed PDA nanospheres, without the need for a reductant or reduction potential.

Here, a porous gold (P-Au) nanomaterial-modified ANE was fabricated by a facile self-assembly method with the help of PDA nanospheres for the determination of $\mathrm{Cu}$ in seawater. The P-Au nanomaterials were self-assembled deposited and immobilized on the ANE surface with partially sacrificed PDA nanospheres. Owing to the outstanding properties of the P-Au nanomaterials and the PDA nanospheres, the fabricated P-Au/PDA-modified ANE (P-Au/PDA/ANE) displays a remarkably improved performance, such as sensitivity and stability for the voltammetric determination of $\mathrm{Cu}$. In addition, the application of the novel P$\mathrm{Au} / \mathrm{PDA} / \mathrm{ANE}$ in real seawater samples for $\mathrm{Cu}$ determination was achieved with satisfactory results.

\section{Experimental}

\subsection{Reagents and materials}

Stainless steel acupuncture needles $(0.25 \times 60 \mathrm{~mm})$ were purchased from Suzhou Medical Appliance Factory Co. Ltd. (China). Silicone rubber (NANDA no. 704) was purchased from Liyang Kangda Chemical Co. Ltd., China. $\mathrm{HAuCl}_{4} \cdot 4 \mathrm{H}_{2} \mathrm{O}$ was purchased from Sinopharm Chemical Reagent Co. Ltd., China, and $2 \mathrm{mM} \mathrm{HAuCl}_{4}$ solution was used for the self-assembly of the $\mathrm{P}-\mathrm{Au}$ nanomaterials. Acetate buffer solution (0.1 M, pH 4.5) was prepared as the supporting electrolyte. All other chemicals were of analytical grade and were used without further purification. Deionized water (18.2 $\mathrm{M} \Omega \mathrm{cm}$ specific resistance) obtained with a Pall Cascada laboratory water system was used throughout the study.

\subsection{Apparatus}

Scanning electron microscope (SEM, Hitachi S-4800 microscope, Japan) was used for the characterization of the morphologies of different ANEs. Element analysis was conducted using an energy dispersive X-ray spectroscope (EDX HORIBAEX-350, Japan). All the electrochemical experiments were conducted on an Electrochemical Work Station (CHI 660E, $\mathrm{CH}$ Instruments, Inc., Shanghai, China). The modified ANE served as the working electrode, $\mathrm{Ag} / \mathrm{AgCl}(3 \mathrm{M} \mathrm{KCl})$ as the reference electrode, and platinum foil as the counter electrode.

\subsection{Preparation of P-Au/PDA-modified ANE}

The schematic for the fabrication process of $\mathrm{P}-\mathrm{Au} / \mathrm{PDA} / \mathrm{ANE}$ and detection of $\mathrm{Cu}^{2+}$ is shown in Scheme 1. Before use, the acupuncture needle was cleaned thoroughly with deionized water. Then, the PDA nanosphere-modified acupuncture needle was obtained by immersing the tip of the cleaned needle in $2 \mathrm{mg} \mathrm{mL}{ }^{-1}$ DA solution (Tris- $\mathrm{HCl}, \mathrm{pH}$ 8.5) with stirring for $8 \mathrm{~h}$. After cleaning with deionized water, the PDA nanospherecovered needle was immersed in a $2 \mathrm{mM} \mathrm{HAuCl}_{4}$ solution with stirring for $8 \mathrm{~h}$ to achieve the self-assembly of P-Au on the sensing tip. After careful cleaning, the P-Au/PDA-modified acupuncture needle was sealed with silicone rubber. During the sealing process, $2 \mathrm{~mm}$ of the needle tip was left for the sensing surface and the needle handle for electrode connection. Eventually, the P-Au/PDA/ANE was obtained after the solidification of the silicone rubber. Similarly, PDA-modified ANE (PDA/ANE) and bare ANE were prepared for comparison. The

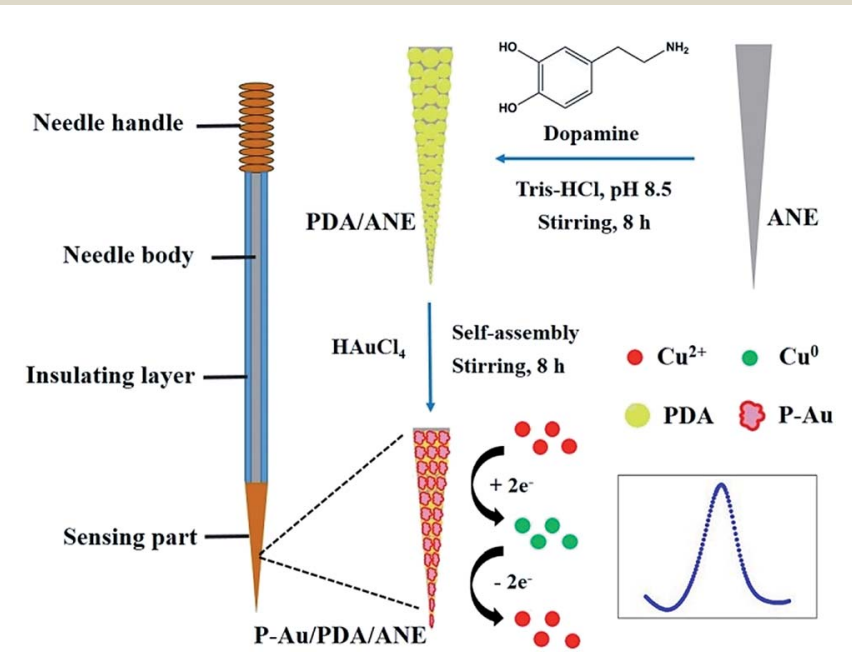

Scheme 1 Schematic for the fabrication process of P-Au/PDA/ANE and detection of $\mathrm{Cu}^{2+}$. 
gold nanomaterial-modified ANE (AuNPs/ANE) was prepared by an electrochemical method (I-T technique, $-0.3 \mathrm{~V}, 120 \mathrm{~s}$ ), considering that it couldn't be fabricated without the PDA nanospheres as a reducing and adhesion agent.

\subsection{Preparation of seawater samples}

The fabricated P-Au/PDA-modified ANE was adopted for the $\mathrm{Cu}$ detection in seawater sampled from Sishili Bay, which belongs to the northern Yellow Sea, China. All the seawater samples were collected into polypropylene containers, which had been washed thoroughly with $1 \mathrm{M} \mathrm{HCl}$ and rinsed with deionized water. After the seawater samples were collected, they were filtrated with $0.45 \mu \mathrm{m}$ membranes, adjusted to $\mathrm{pH} 1.8$ with $\mathrm{HCl}$, and stored in polyethylene bottles at $4{ }^{\circ} \mathrm{C}$ until use.

\subsection{Electrochemical analysis procedure}

The electrochemical impedance spectra (EIS) of different ANEs were conducted in a $0.1 \mathrm{M} \mathrm{KCl}$ solution with $5 \mathrm{mM} \mathrm{K}_{3}\left[\mathrm{Fe}(\mathrm{CN})_{6}\right]$. Except for the $\mathrm{Cu}$ detection in real seawater samples, the cyclic voltammetry $(\mathrm{CV})$ and square wave anodic stripping voltammetry (SWASV) experiments were conducted in an acetate buffer (pH 4.5) solution. The anodic stripping voltammograms for the determination of $\mathrm{Cu}$ were obtained by applying SWASV scans in an acetate buffer or real sweater samples. During the accumulation step, $-0.3 \mathrm{~V}$ and $120 \mathrm{~s}$ were adopted as the accumulation potential and time, respectively. For the SWASV scans, -0.1 to $0.5 \mathrm{~V}, 0.025 \mathrm{~V}$, and $25 \mathrm{~Hz}$ and were used as the scan range, amplitude, and frequency, respectively. A cleaning procedure was applied before the next measurement with a potential of $0.4 \mathrm{~V}$ and a time of $30 \mathrm{~s}$ as the parameters. The standard addition method was adopted for $\mathrm{Cu}$ determination in the seawater samples.

\section{Results and discussion}

\subsection{Characterization of P-Au/PDA-modified ANE}

To investigate the surface morphology of P-Au/PDA/ANE, SEM characterization was conducted (Fig. 1). It can be clearly seen that the surface of the bare ANE is very smooth and the needle tip diameter is about $5 \mu \mathrm{m}$ (Fig. 1A). It can be expected that the smooth surface will provide few binding sites for the combination of functional nanomaterials. For the PDA/ANE (Fig. 1B), PDA nanospheres with a diameter of about 100-200 nm were obtained on the ANE surface after the self-polymerization of dopamine (DA). The PDA nanospheres covered on ANE could enhance the specific surface area, provide more binding sites, and contribute as a reductant for $\mathrm{HAuCl}_{4}$, and as an adhesion agent to immobilize the gold nanomaterials. As for the P-Au/ PDA/ANE, it can be seen that the gold nanomaterials that cover the PDA/ANE surface with a porous structure (Fig. 1C and D). The PDA nanospheres were partially consumed while reducing $\mathrm{HAuCl}_{4}$ to $\mathrm{Au}^{0}$, and the formed gold nanomaterials covered the partially sacrificed PDA nanospheres to form the porous structure. More simply, the partially sacrificed PDA nanospheres might have acted as the template for the formation of gold nanomaterials with a porous structure. It is not easy
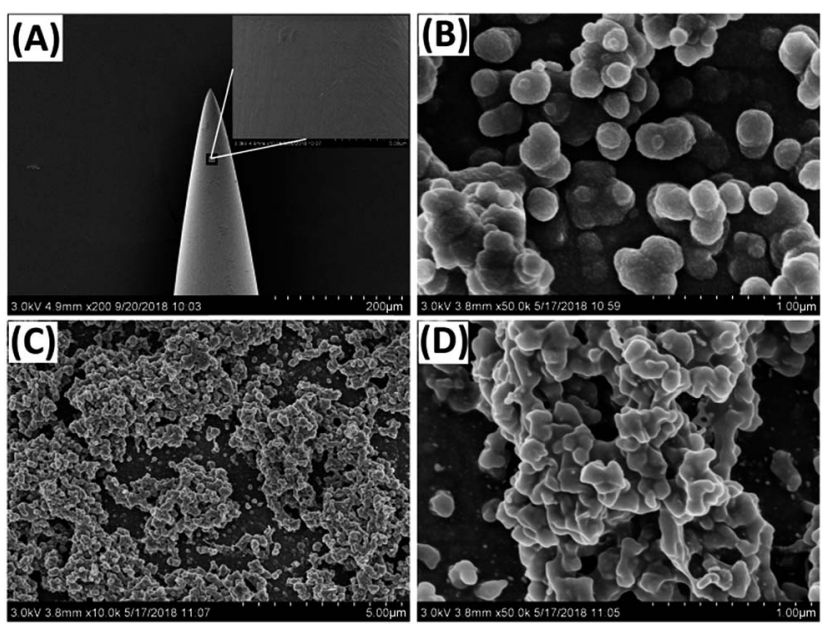

Fig. 1 SEM images of the bare ANE (A), PDA/ANE (B), and P-Au/PDA/ ANE (C and D).

to distinguish the rest of the PDA nanospheres from the SEM images of $\mathrm{P}-\mathrm{Au} / \mathrm{PDA} / \mathrm{ANE}$ due to the P-Au nanomaterials covering the surface. Considering the adhesion capability of PDA, there is no need to additionally cover protective layer on the P-Au nanomaterial surface to prevent its peel off. Owing to the outstanding electrocatalytic and conductive capabilities, the fabricated P-Au/PDA/ANE displayed a remarkably improved electrochemical response for the voltammetric determination of copper.

According to the producer, the acupuncture needles were made of $06 \mathrm{Cr}_{19} \mathrm{Ni}_{10}$ stainless steel. To verify the composition of acupuncture needles and existence of PDA nanospheres and P$\mathrm{Au}$ nanomaterials, EDS was conducted to investigate the elemental composition of the fabricated P-Au/PDA/ANE (Fig. 2). The EDS pattern of the bare ANE shows that $\mathrm{Fe}, \mathrm{Cr}$, and Ni were the major elements forming the acupuncture needle made of stainless steel. Except for $\mathrm{Fe}, \mathrm{Cr}$, and Ni, the EDS pattern of PDA/ ANE exhibits $\mathrm{C}$ element at about $0.26 \mathrm{keV}$, which proves the

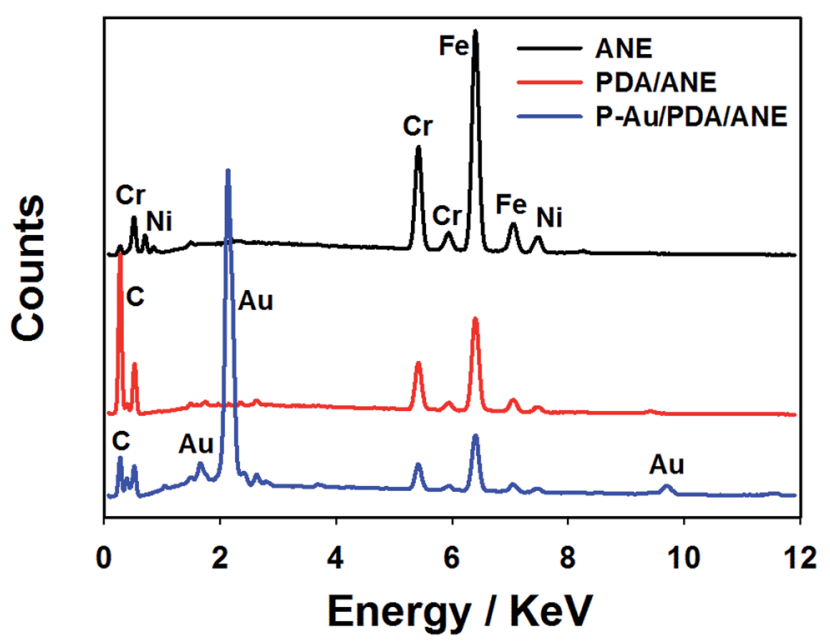

Fig. 2 EDS patterns of the bare ANE, PDA/ANE, and P-Au/PDA/ANE. 
presence of the PDA nanospheres. After the assembly of the $\mathrm{P}$-Au nanomaterials on PDA/ANE, three typical Au peaks appear at about $2.15 \mathrm{keV}, 1.65 \mathrm{keV}$, and $9.70 \mathrm{keV}$ and a $\mathrm{C}$ peak in the EDS pattern of P-Au/PDA/ANE, which proves the existence of the $\mathrm{P}-\mathrm{Au}$ nanomaterials and PDA nanospheres. The successful fabrication of the P-Au/PDA-modified ANE can be demonstrated by the results of the SEM and EDS characterizations.

\subsection{Electrochemical behaviours of the P-Au/PDA-modified} ANE

Electrochemical techniques like $\mathrm{CV}$ and EIS were adopted to investigate the electrochemical behavior of the fabricated P-Au/ PDA/ANE. The typical CV curves of the bare ANE, PDA/ANE, and $\mathrm{P}-\mathrm{Au} / \mathrm{PDA} / \mathrm{ANE}$ are shown in Fig. 3. As expected, the two CV curves of the bare ANE and PDA/ANE are very similar, and no peak can be observed. It can be concluded that the modification of PDA nanospheres has no obvious effect on the electrochemical properties of the bare ANE. As for the P-Au/PDA/ANE, a typical peak of the gold nanomaterial appears at about $0.63 \mathrm{~V}^{32}$ which further proves the existence of the P-Au nanomaterials. More importantly, the self-assembly of the P-Au nanomaterial on the sensing surface increases the background current signal significantly, which might be caused by their excellent conductivity.

As an effective tool for the evaluation of electrochemically active surfaces, EIS was adopted to verify the effects of the P-Au nanomaterials and PDA nanospheres on the electron transfer capability (Fig. 4). The EIS of the $\mathrm{Fe}(\mathrm{CN})_{6}{ }^{3-} / \mathrm{Fe}(\mathrm{CN})_{6}{ }^{4-}$ electron transfer reaction on the bare ANE, PDA/ANE, and P-Au/PDA/ANE are in agreement with the standard Randles equivalent model circuit (inset in Fig. 4). The equivalent circuit includes the solution resistance $\left(R_{\mathrm{S}}\right)$, the charge transfer resistance $\left(R_{\mathrm{ct}}\right)$, the constant phase element (CPE), and the Warburg impedance $\left(Z_{\mathrm{w}}\right)$. It is known that the semicircle diameter of EIS obtained in the high frequency part corresponds to the charge-transfer limited electrochemical processes. The diameter of the semicircle is equal to the value of the $R_{\mathrm{ct}}$ in the Nyquist plot, which

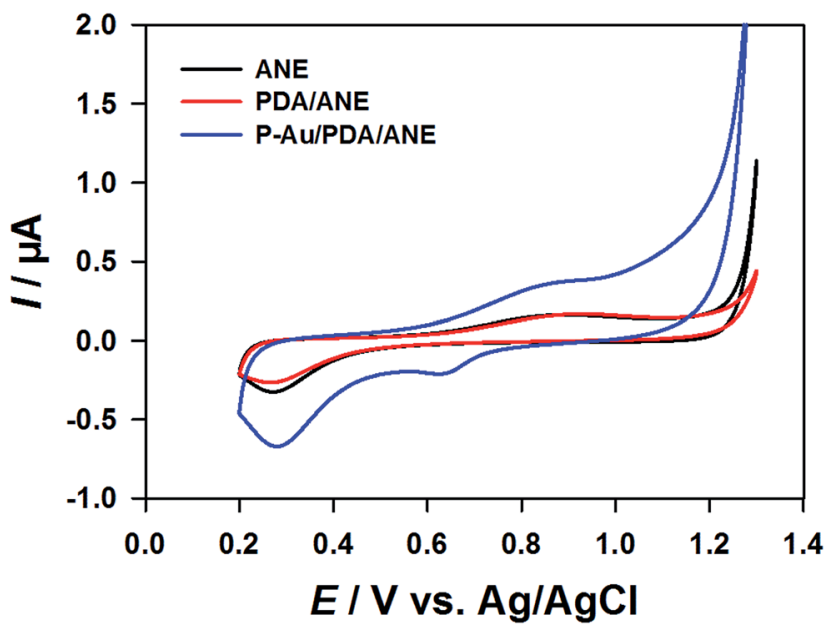

Fig. 3 The CV curves of the bare ANE, PDA/ANE, and P-Au/PDA/ANE in acetate buffer solution (0.1 M, pH 4.5).

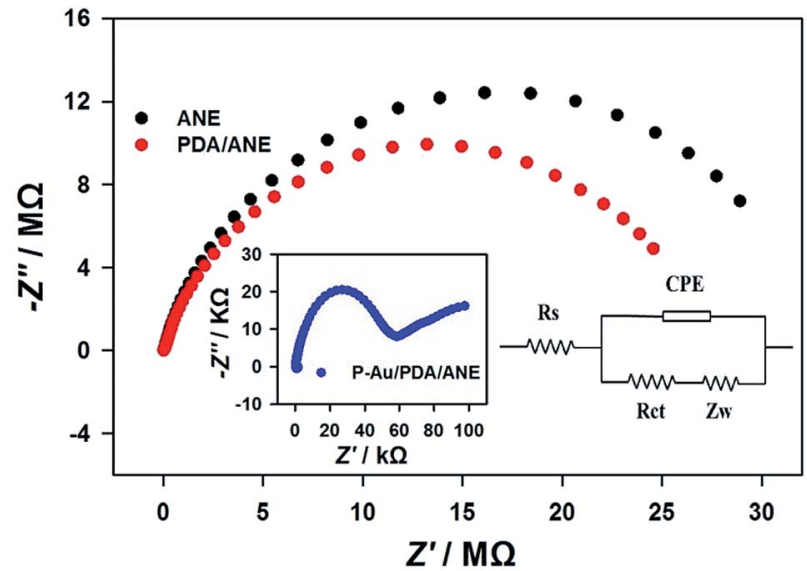

Fig. 4 Electrochemical impedance spectra and equivalent circuit of the bare ANE, PDA/ANE, and P-Au/PDA/ANE obtained in $0.1 \mathrm{M} \mathrm{KCl}$ solution with $5 \mathrm{mM} \mathrm{K}_{3}\left[\mathrm{Fe}(\mathrm{CN})_{6}\right]$.

controls the electron transfer kinetics of the redox probe on the electrode surface. After covering the ANE with the PDA nanospheres, the diameter of the semicircle showed no obvious change, and from this result, it can be concluded that the PDA nanospheres have almost no effect on the electron transfer of the redox probe. The $R_{\mathrm{ct}}$ of the bare ANE was calculated as 3.27 $\times 10^{7} \Omega$, which confirms that the charge transfer process is very slow on the bare ANE. After the modification of the ANE surface with PDA nanospheres, the $R_{\text {ct }}$ decreased slightly to $2.83 \times$ $10^{7} \Omega$. Therefore, the modification of the PDA nanospheres has almost no influence on the conductivity of the ANE. As expected, after the modification of the P-Au nanomaterials on the electrode surface, the $R_{\mathrm{ct}}$ of P-Au/PDA/ANE decreased remarkably to $5.24 \times 10^{4} \Omega$, which was much smaller than that of the PDA/ ANE and the bare ANE. In other words, the conductivity of the electrode improved significantly after the modification of P-Au nanomaterials. Through the comparison of different ANEs on the EIS, it can be concluded that the decrease in the $R_{\mathrm{ct}}$ of P-Au/ PDA/ANE is mainly caused by the P-Au nanomaterials, which have an outstanding ability in promoting the electron transfer. So, the P-Au/PDA/ANE has been successfully fabricated and $\mathrm{P}$-Au nanomaterials have the excellent capability for facilitating the electron transfer and improving the conductivity of the electrode.

\subsection{Voltammetric response of $\mathrm{Cu}^{2+}$ on the P-Au/PDA- modified ANE}

SWASV was adopted to investigate the voltammetric response of $\mathrm{Cu}^{2+}$ on different ANEs (Fig. 5). It can be clearly seen that no stripping current signal could be obtained using the bare ANE for the determination of $50 \mathrm{nM}$ of $\mathrm{Cu}^{2+}$. Moreover, the voltammetric response for $50 \mathrm{nM} \mathrm{Cu}{ }^{2+}$ obtained on the PDA/ANE is very similar to that of the bare ANE. This phenomenon indicates that the modification of the PDA nanospheres on the ANE has almost no effect on the voltammetric determination of $\mathrm{Cu}^{2+}$. Interestingly, when the P-Au/PDA/ANE is used, a large oxidation peak caused by the oxidation process of $\mathrm{Cu}$ to $\mathrm{Cu}^{2+}$ appears at 


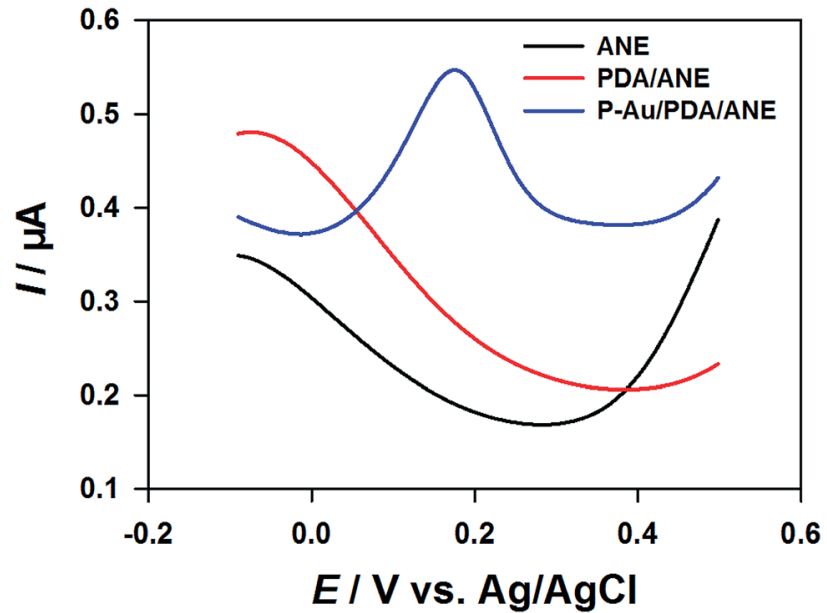

Fig. 5 SWASV curves of the bare ANE, PDA/ANE, and P-Au/PDA/ANE for $50 \mathrm{nM} \mathrm{Cu}^{2+}$.

about $0.18 \mathrm{~V}$. Through the comparison of the performances of different ANEs for the $\mathrm{Cu}^{2+}$ determination, it can be concluded that the P-Au/PDA/ANE shows an enhanced performance due to its excellent electrocatalytic activity and the conductivity of the $\mathrm{P}-\mathrm{Au}$ nanomaterials. When compared with electrodes modified using other aromatic compound-based layers, the modification of PDA nanospheres has almost no improvement on the voltammetric determination of $\mathrm{Cu}^{2+} \cdot{ }^{37,38}$ However, it should be noted that although PDA nanospheres have no promoting effect, they are helpful for the formation of gold nanomaterials with a porous structure, which have an excellent electrocatalytic activity.

\subsection{Optimization of $\mathrm{Cu}^{2+}$ determination on P-Au/PDA- modified ANE}

To obtain the best response for $\mathrm{Cu}^{2+}$ determination on $\mathrm{P}-\mathrm{Au} /$ PDA/ANE, the parameters including the accumulation potential and time have been optimized. Different potentials from $0.1 \mathrm{~V}$ to $-0.7 \mathrm{~V}$ (accumulation time fixed as $120 \mathrm{~s}$ ) were applied during the accumulation process to investigate their influence on the current response of $\mathrm{Cu}^{2+}$ (Fig. 6A). It can be seen that the peak current $\left(I_{\mathrm{p}}\right)$ increases gradually when the potential decreases from $0.1 \mathrm{~V}$ to $-0.3 \mathrm{~V}$ and reaches a maximum value at $-0.3 \mathrm{~V}$. In this process, more $\mathrm{Cu}^{2+}$ could be reduced and accumulated on the P-Au/PDA/ANE surface by more negative potentials. However, the $I_{\mathrm{p}}$ decreases when the potential continues to decrease from $-0.3 \mathrm{~V}$ to $-0.7 \mathrm{~V}$. In this potential range, the dissolved oxygen in solution would compete with $\mathrm{Cu}^{2+}$ to obtain the elsectrons, which decreases the accumulation amount of $\mathrm{Cu}$ on the electrode surface and the corresponding stripping current. ${ }^{25}$ So, $-0.3 \mathrm{~V}$ was chosen and used as the accumulation potential in this study.

After the accumulation potential was adopted and fixed at $-0.3 \mathrm{~V}$, the accumulation time ranging from $30 \mathrm{~s}$ to $360 \mathrm{~s}$ was applied to investigate its effect on the current response (Fig. 6B). It can be clearly observed that the $I_{\mathrm{p}}$ increases with the accumulation time ranging from $30 \mathrm{~s}$ to $270 \mathrm{~s}$, and the
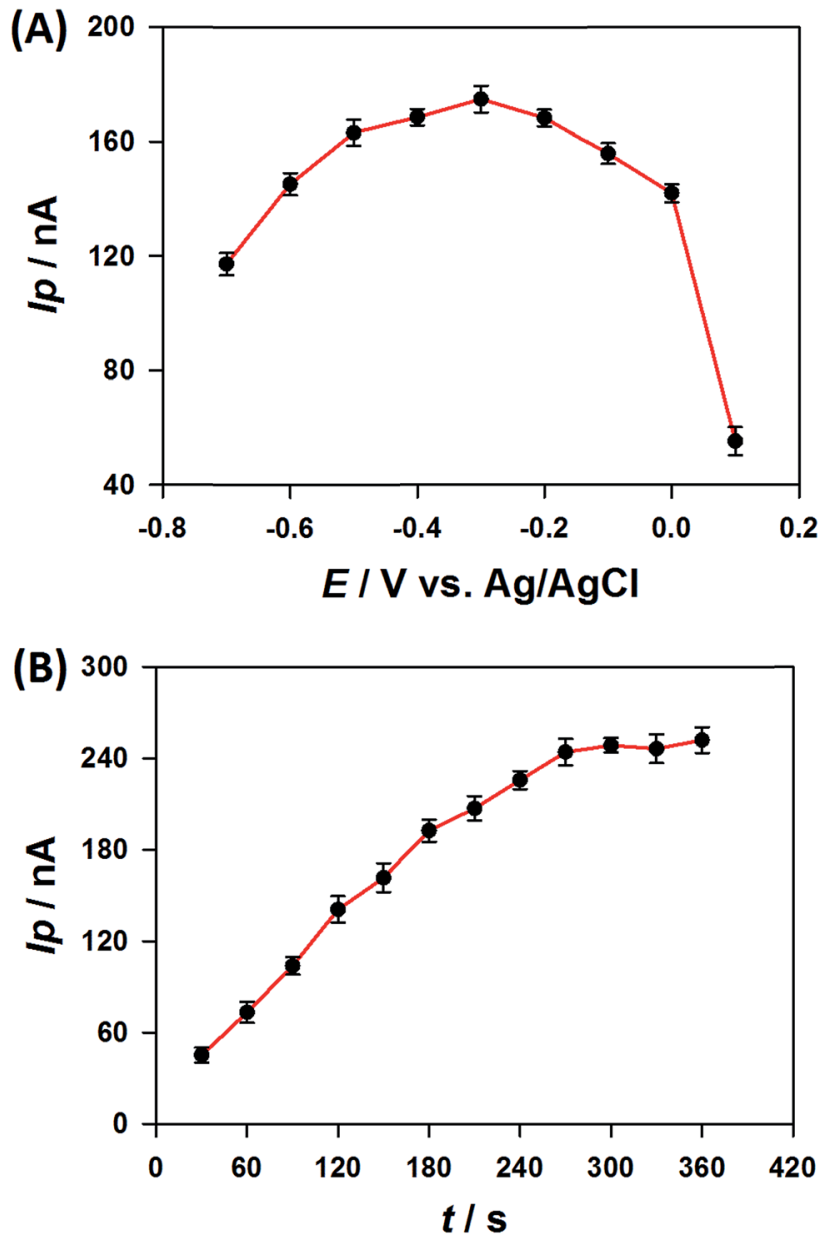

Fig. 6 The peak current $\left(I_{\mathrm{p}}\right)$ obtained at P-Au/PDA/ANE for $50 \mathrm{nM}$ $\mathrm{Cu}^{2+}$ with different experimental parameters: accumulation potential (A) and accumulation time (B) in acetate buffer ( $\mathrm{pH} 4.5)$ solution.

increasing trend tends to be slower when the time increases to more than $270 \mathrm{~s}$. It is known that more $\mathrm{Cu}^{2+}$ will be adsorbed, reduced and accumulated on the electrode surface with an increase in the accumulation time, which leads to the an increase in $I_{\mathrm{p}}$. After the accumulation time reaching $270 \mathrm{~s}$, the slowdown of the increasing trend of $I_{\mathrm{p}}$ might be caused by the adsorption equilibrium between $\mathrm{Cu}^{2+}$ and electrode surface. In this study, $120 \mathrm{~s}$ was adopted as the accumulation time, which is adequate for the determination of $\mathrm{Cu}$ in seawater samples. It should be noted that a longer accumulation time could be applied if a higher sensitivity is needed.

\subsection{Analytical performance of P-Au/PDA-modified ANE}

To investigate the analytical performance of P-Au/PDA/ANE, the calibration curve for $\mathrm{Cu}^{2+}$ determination was derived from the SWASV curves obtained in acetate buffer $(\mathrm{pH} 4.5)$ solution (Fig. 7). The $I_{\mathrm{p}}$ increases linearly with the $\mathrm{Cu}^{2+}$ concentration ranging from $0.7 \mathrm{nM}$ to $1000 \mathrm{nM}$, and the equation for linear regression is $I_{\mathrm{p}}=2.60 \mathrm{C}+0.01$ with a correlation coefficient of 0.997 . The detection limit of $\mathrm{P}-\mathrm{Au} /$ PDA/ANE for $\mathrm{Cu}^{2+}$ determination was calculated as $0.24 \mathrm{nM}$ 

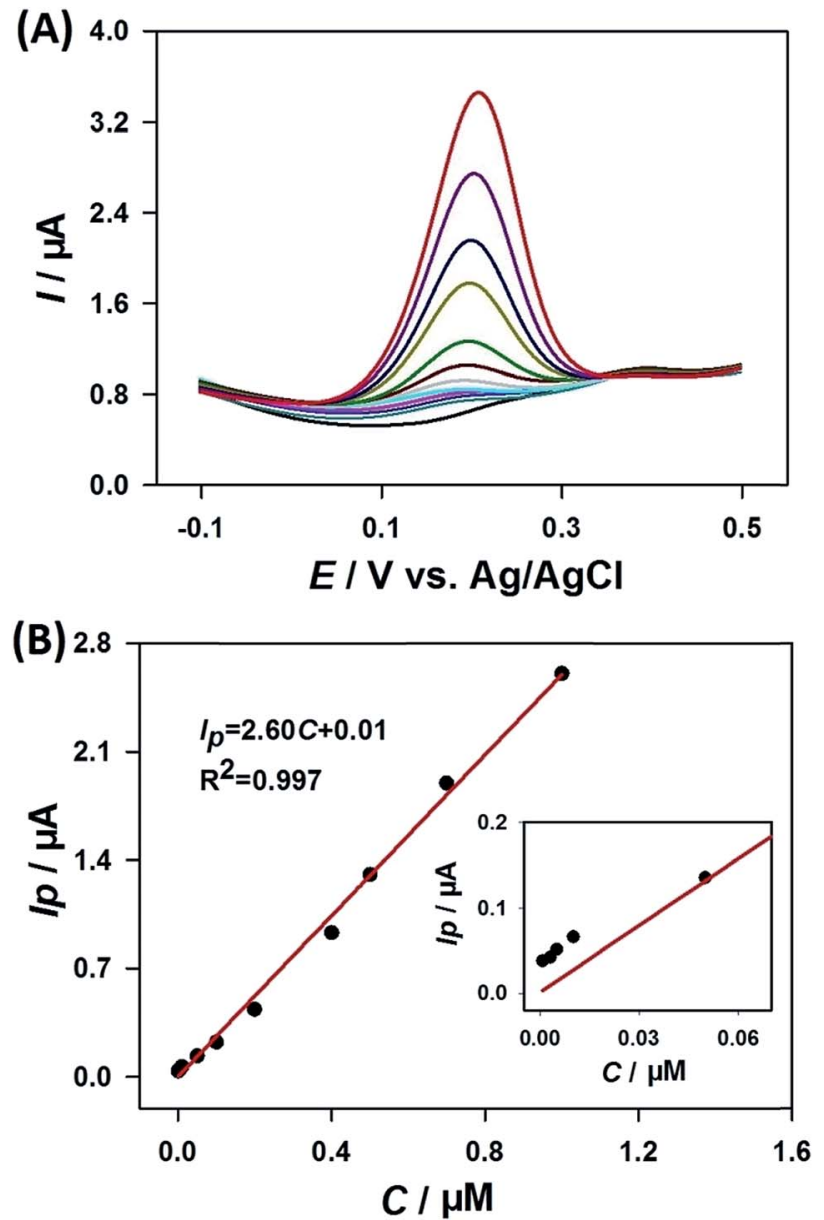

Fig. 7 SWASV curves (A) and corresponding calibration curve (B) of P$\mathrm{Au} / \mathrm{PDA} / \mathrm{ANE}$ with successive addition of $0 \mathrm{nM}, 0.7 \mathrm{nM}, 3 \mathrm{nM}, 5 \mathrm{nM}$, $10 \mathrm{nM}, 50 \mathrm{nM}, 100 \mathrm{nM}, 200 \mathrm{nM}, 400$ nM, 500 nM, 700 nM, $1000 \mathrm{nM}$ $\mathrm{Cu}^{2+}$ (from bottom to top) to acetate buffer $(\mathrm{pH} 4.5)$ solution.

with the accumulation potential and time as $-0.3 \mathrm{~V}$ and $120 \mathrm{~s}$, respectively. It should be noted that if a lower limit of detection needed, Nafion, one of the most widely used cationexchange polymers, can be coated on the P-Au nanomaterial surface as an adsorption agent to further improve the sensitivity. ${ }^{32}$ Stainless steel electrode $\left(1 \times 1 \mathrm{~cm}^{2}\right)$ has been previously adopted and modified with functional nanomaterials for the electrochemical determination of $\mathrm{Cu}, \mathrm{Pb}$ and $\mathrm{Hg}^{39}$ Although only $\mathrm{Cu}$ was detected here, the P-Au/PDA/ANE shows a lower detection limit, which might be caused by the small size and unique aciform structure of ANE and the excellent electrocatalytic activity of P-Au. In addition, Table 1 shows the comparison of P-Au/PDA/ANE with other electrodes previously proposed for the determination of $\mathrm{Cu}^{2+}$. The results show that when compared with other electrodes, the newly fabricated P-Au/PDA/ANE is easy to construct and exhibits a wider linear range and a lower limit of detection. Above all, considering its satisfactory performance, the so-fabricated P$\mathrm{Au} / \mathrm{PDA} / \mathrm{ANE}$ might be a good candidate for the $\mathrm{Cu}$ determination in seawater samples.
Table 1 Comparison of the P-Au/PDA/ANE with previously reported electrodes for $\mathrm{Cu}^{2+}$ determination

\begin{tabular}{|c|c|c|c|c|}
\hline Electrodes $^{a}$ & Methods $^{b}$ & $\begin{array}{l}\text { Linear } \\
\text { range (nM) }\end{array}$ & $\begin{array}{l}\text { Detection } \\
\text { limit (nM) }\end{array}$ & Ref. \\
\hline $\mathrm{rGO} / \mathrm{Bi} / \mathrm{CPE}$ & DPASV & $300-1600$ & 400 & 11 \\
\hline CNT/Nafion/GCE & LSSV & $100-900$ & 51 & 12 \\
\hline L-Cys-rGO/GCE & DPASV & $400-2000$ & 40 & 40 \\
\hline $\begin{array}{l}\text { Sn/AuNPs/gold } \\
\text { microelectrode }\end{array}$ & SWASV & $80-8000$ & 30 & 41 \\
\hline Ph-GO/gold electrode & OSWV & $300-1600$ & 27 & 42 \\
\hline Hg/MWCNTs/CHIT/SPE & SWASV & $40-200$ & 12 & 14 \\
\hline $\mathrm{SA} / \mathrm{SWNTs} / \mathrm{rGO} / \mathrm{GCE}$ & DPASV & $200-2000$ & 6 & 43 \\
\hline $\mathrm{Au} @ \mathrm{MnO}_{2} / \mathrm{GCE}$ & DPASV & $20-1000$ & 4.9 & 1 \\
\hline $\mathrm{P}-\mathrm{Au} / \mathrm{PDA} / \mathrm{ANE}$ & SWASV & $0.7-1000$ & 0.24 & $\begin{array}{l}\text { This } \\
\text { work }\end{array}$ \\
\hline
\end{tabular}

${ }^{a} \mathrm{rGO} / \mathrm{Bi} / \mathrm{CPE}$ : reduced graphene oxide/bismuth nanocompositemodified carbon paste electrode, CNT/Nafion/GCE: carbon nanotubes-/Nafion-modified glass carbon electrode, L-Cys-rGO/GCE: Lcysteine-reduced graphene oxide-modified glass carbon electrode, $\mathrm{Sn} /$ AuNPs/gold microelectrode: tin film/gold nanoparticle-modified gold microelectrode, $\mathrm{Ph}$-GO/gold electrode: 4 -aminophenyl/graphene oxidemodified gold electrode, $\mathrm{Hg} / \mathrm{MWCNTs} / \mathrm{CHIT} / \mathrm{SPE}$ : mercury nanodroplets/multi-walled carbon nanotubes-/chitosan-modified screenprinted electrode, SA/SWNTs/rGO/GCE: sodium alginate/single-walled carbon nanotubes/reduced graphene oxide-modified glass carbon electrode, $\mathrm{Au} @ \mathrm{MnO}_{2} / \mathrm{GCE}$ : gold@ $\mathrm{MnO}_{2}$-modified glass carbon electrode. ${ }^{b}$ DPASV: differential pulse anodic stripping voltammetry, LSSVL: linear sweep stripping voltammetry, OSWV: Osteryoung square wave voltammetry.

\subsection{Reproducibility, repeatability and selectivity}

To investigate the suitability of the P-Au/PDA/ANE for $\mathrm{Cu}$ determination in complex seawater samples, its repeatability, reproducibility, and stability were all evaluated. The reproducibility of the P-Au/PDA/ANE was investigated by determining $50 \mathrm{nM} \mathrm{Cu}^{2+}$ in acetate buffer ( $\left.\mathrm{pH} 4.5\right)$ solution with six independently prepared electrodes and the relative standard deviation (RSD) was calculated as $3.6 \%$. The repeatability was evaluated by determining $50 \mathrm{nM} \mathrm{Cu}^{2+}$ with the same P-Au/PDA/ ANE for twenty measurements, and the RSD was calculated as $1.3 \%$. Hence, the novel P-Au/PDA/ANE fabricated here has a good reproducibility and repeatability performance. As for the stability, after the P-Au/PDA/ANE was fabricated, it was tested by determining $\mathrm{Cu}^{2+}$ for three measurements each day and the current response had no obvious decrease after six days (retaining $94 \%$ of the initial value). For comparison, the stability of the AuNPs/ANE was tested in the same way and the current response decreased significantly during four days. The improved stability of the P-Au/PDA/ANE might be caused by the strong adhesion between the P-Au nanomaterials and the ANE induced by the partially sacrificed polydopamine nanospheres due to their excellent adhesion capability.

There is no doubt that a good selectivity is needed for the accurate determination of $\mathrm{Cu}$ in real seawater samples considering the presence of large amounts of interfering substances. To investigate the anti-interference ability of the P-Au/PDA/ANE, various possible interfering species were added into the acetate buffer solution with $50 \mathrm{nM} \mathrm{\textrm {Cu } ^ { 2 + }}$ under the optimal working 


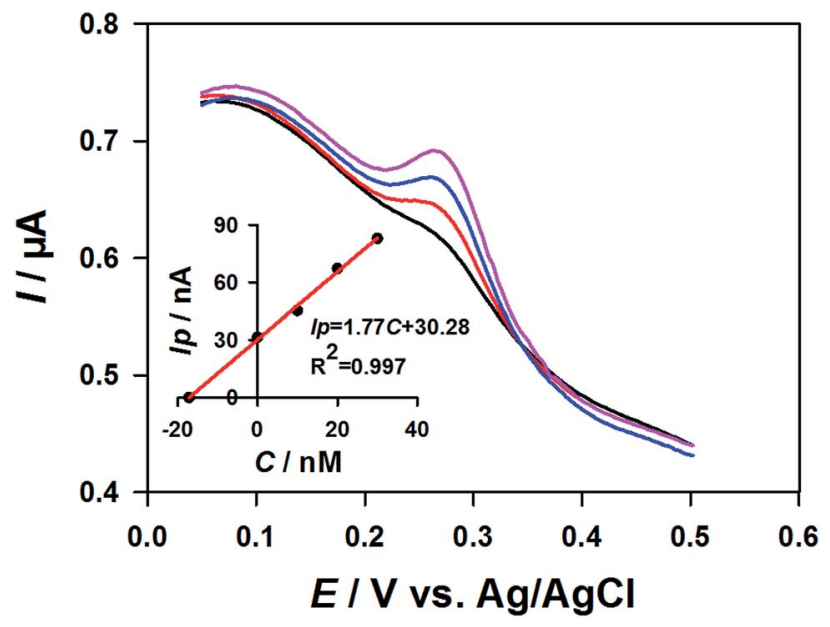

Fig. 8 SWASV curves obtained with the P-Au/PDA/ANE in seawater sample with successive addition of $0 \mathrm{nM}, 10 \mathrm{nM}$, and $20 \mathrm{nM} \mathrm{Cu}^{2+}$ (from bottom to top), and the corresponding linear regression equation.

Table 2 The results obtained with the P-Au/PDA/ANE for real seawater samples determination

Seawater samples $\quad \mathrm{Cu}^{2+}$ added $(\mathrm{nM}) \quad$ Mean $(\mathrm{nM}) \pm \mathrm{SD} \quad$ Recovery (\%)

\begin{tabular}{llrl}
\hline & & & \\
Seawater 1 & 0 & $19.46 \pm 0.18$ & - \\
Seawater 2 & 0 & $17.16 \pm 0.35$ & - \\
Seawater 3 & 0 & $26.88 \pm 0.80$ & - \\
& 10 & $37.56 \pm 1.05$ & 108.97 \\
& 20 & &
\end{tabular}

conditions. The results showed that addition of a 100-fold concentration of cations such as $\mathrm{Mg}^{2+}, \mathrm{Zn}^{2+}, \mathrm{Ni}^{2+}$, and $\mathrm{Co}^{2+}$ and anions such as $\mathrm{SO}_{4}{ }^{2-}$ and $\mathrm{NO}_{3}{ }^{-}$had no influence on $\mathrm{Cu}^{2+}$ detection $\left(<5 \%\right.$ current change). The addition of 50 -fold $\mathrm{Cd}^{2+}$, $\mathrm{Fe}^{3+}$, and 10 -fold $\mathrm{Bi}^{3+}, \mathrm{Pb}^{2+}$ also had no obvious effect on the determination of $\mathrm{Cu}^{2+}$. The data images of reproducibility, repeatability and selectivity are shown in Fig. S1. $\dagger$ Thus, our newly fabricated P-Au/PDA/ANE has a good anti-interference ability and great potential for $\mathrm{Cu}$ determination in seawater samples.

\subsection{Analytical applications}

To investigate the practical application of the P-Au/PDA/ANE, it was used for the determination of $\mathrm{Cu}$ in real seawater samples collected from Sishili Bay, China. The contents of $\mathrm{Cu}$ in different seawater samples were quantified by the standard addition method. Fig. 8 shows the typical SWASV curves obtained with the P-Au/PDA/ANE in a seawater sample with the successive addition of $0 \mathrm{nM}, 10 \mathrm{nM}$, and $20 \mathrm{nM}$ of $\mathrm{Cu}^{2+}$ and the corresponding linear regression equation. The $\mathrm{Cu}$ concentration in this seawater sample has been calculated to be $17.16 \mathrm{nM}$. Table 2 shows the concentrations of $\mathrm{Cu}^{2+}$ obtained by P-Au/PDA/ ANE and corresponding recovery in these samples. The results indicate that the P-Au/PDA/ANE fabricated here is reliable and suitable for the determination $\mathrm{Cu}$ in seawater.

\section{Conclusions}

In this study, a P-Au/PDA-functionalized ANE was fabricated by a facile self-assembly method for the voltammetric determination of copper in seawater. The PDA nanospheres coated on the sensing part of the ANE by a self-polymerization method played an important role in the formation and immobilization of the PAu nanomaterials. The P-Au nanomaterials were self-assembled and deposited on the ANE with the reduction of $\mathrm{AuCl}_{4}{ }^{-}$by PDA nanospheres which were gradually sacrificed. Owing to the unique nanostructure and electrocatalytic ability of the P-Au nanomaterials, the fabricated P-Au/PDA/ANE displayed an excellent anodic stripping voltammetric response for $\mathrm{Cu}$ determination. Moreover, the stability of the modified ANE was improved significantly due to the strong adhesion stability of PDA and no protective layer was needed. Furthermore, the successful application of the P-Au/PDA/ANE in real seawater samples for the determination of $\mathrm{Cu}$ was obtained via SWASV.

\section{Conflicts of interest}

There are no conflicts to declare.

\section{Acknowledgements}

This work was financially supported by the Key Research and Development Plan of Yantai City (2018ZHGY076), the Key Research and Development Plan of Shandong Province (2017GHY215002), and the Key Research and Development Plan of Yantai City (2017ZH096).

\section{References}

1 H. Wei, D. Pan, X. Hu, M. Liu, H. Han and D. Shen, Microchim. Acta, 2018, 185, 258.

2 G. Özzeybek, S. Erarpat, D. S. Chormey, M. Firat, Ç. Büyükpınar, F. Turak and S. Bakırdere, Microchem. J., 2017, 132, 406-410.

3 V. Yilmaz, Z. Arslan, O. Hazer and H. Yilmaz, Microchem. J., 2014, 114, 65-72.

4 M. Liu, D. Pan, W. Pan, Y. Zhu, X. Hu, H. Han, C. Wang and D. Shen, Talanta, 2017, 174, 500-506.

5 G. J. Brewer, Chem. Res. Toxicol., 2010, 23, 319-326.

6 P.-L. Lee, Y.-C. Sun and Y.-C. Ling, J. Anal. At. Spectrom., 2009, 24, 320-327.

7 S. Su, B. Chen, M. He and B. Hu, Talanta, 2014, 123, 1-9.

8 J. Vukovic, S. Matsuoka, K. Yoshimura, V. Grdinic, R. J. Grubesic and O. Zupanic, Talanta, 2007, 71, 2085-2091.

9 R. Zhang, M. Peng, C. Zheng, K. Xu and X. Hou, Microchem. J., 2016, 127, 62-67.

10 L. N. Santos, J. A. G. Neto and N. M. Caldas, Fuel, 2012, 99, 912.

11 P. K. Sahoo, B. Panigrahy, S. Sahoo, A. K. Satpati, D. Li and D. Bahadur, Biosens. Bioelectron., 2013, 43, 293-296.

12 J. J. Silva, L. L. Paim and N. R. Stradiotto, Electroanalysis, 2014, 26, 1794-1800. 
13 A. Afkhami, M. Soltani-Shahrivar, H. Ghaedi and T. Madrakian, Electroanalysis, 2016, 28, 296-303.

14 W. Song, L. Zhang, L. Shi, D.-W. Li, Y. Li and Y.-T. Long, Microchim. Acta, 2010, 169, 321-326.

15 A. Annibaldi, S. Illuminati, C. Truzzi and G. Scarponi, Mar. Pollut. Bull., 2011, 62, 2813-2821.

16 A. Annibaldi, S. Illuminati, C. Truzzi, G. Libani and G. Scarponi, Mar. Pollut. Bull., 2015, 96, 441-449.

17 V. Vyskočil and J. Barek, Crit. Rev. Anal. Chem., 2009, 39, 173188.

18 L. Yang, Y. Li, Y. Zhang, D. Fan, X. Pang, Q. Wei and B. Du, ACS Appl. Mater. Interfaces, 2017, 9, 35260-35267.

19 X. Ren, H. Ma, T. Zhang, Y. Zhang, T. Yan, B. Du and Q. Wei, ACS Appl. Mater. Interfaces, 2017, 9, 37637-37644.

20 X. Pang, C. Cui, M. Su, Y. Wang, Q. Wei and W. Tan, Nano Energy, 2018, 46, 101-109.

21 X. Ren, P. Lu, R. Feng, T. Zhang, Y. Zhang, D. Wu and Q. Wei, Talanta, 2018, 188, 593-599.

22 J.-X. Zhou, L.-N. Tang, F. Yang, F.-X. Liang, H. Wang, Y.-T. Li and G.-J. Zhang, Analyst, 2017, 142, 4322-4329.

23 X. Niu, Z. Wen, X. Li, W. Zhao, X. Li, Y. Huang, Q. Li, G. Li and W. Sun, Sens. Actuators, B, 2018, 255, 471-477.

24 Y. Zhu, D. Pan, X. Hu, H. Han, M. Lin and C. Wang, Sens. Actuators, B, 2017, 243, 1-7.

25 H. Han, D. Pan, C. Wang and R. Zhu, RSC Adv., 2017, 7, 15833-15841.

26 H. Han, D. Pan, D. Liu, X. Hu, M. Lin and F. Li, Frontiers in Marine Science, 2015, 2, 75.

27 D. Gloria, J. Justin Gooding, G. Moran and D. Brynn Hibbert, J. Electroanal. Chem., 2011, 656, 114-119.

28 K. Sakai, Y. Kitazumi, O. Shirai and K. Kano, Anal. Sci., 2018, 34, 1317-1322.
29 X. Ren, T. Zhang, D. Wu, T. Yan, X. Pang, B. Du, W. Lou and Q. Wei, Biosens. Bioelectron., 2017, 94, 694-700.

30 L. Yang, W. Zhu, X. Ren, M. S. Khan, Y. Zhang, B. Du and Q. Wei, Biosens. Bioelectron., 2017, 91, 842-848.

31 X. Ren, J. Yan, D. Wu, Q. Wei and Y. Wan, ACS Sens., 2017, 2, 1267-1271.

32 H. Han, W. Tao, X. Hu, X. Ding, D. Pan, C. Wang and S. Xu, Electrochim. Acta, 2018, 289, 474-482.

33 S. Kim, L. K. Jang, H. S. Park and J. Y. Lee, Sci. Rep., 2016, 6, 30475.

34 P. Glass, H. Chung, N. R. Washburn and M. Sitti, Langmuir, 2009, 25, 6607-6612.

35 A.-J. Wang, Q.-C. Liao, J.-J. Feng, Z.-Z. Yan and J.-R. Chen, Electrochim. Acta, 2012, 61, 31-35.

36 X. Zheng, Y. Guo, J. Zheng, C. Ma, X. Zhou, J. Lin and R. Lin, Russ. J. Electrochem., 2017, 53, 443-451.

37 Y. Oztekin, Z. Yazicigil, A. Ramanaviciene and A. Ramanavicius, Sens. Actuators, B, 2011, 152, 37-48.

38 Y. Oztekin, M. Tok, H. Nalvuran, S. Kiyak, T. Gover, Z. Yazicigil, A. Ramanaviciene and A. Ramanavicius, Electrochim. Acta, 2010, 56, 387-395.

39 M. A. Deshmukh, R. Celiesiute, A. Ramanaviciene, M. D. Shirsat and A. Ramanavicius, Electrochim. Acta, 2018, 259, 930-938.

40 S. Muralikrishna, K. Sureshkumar, T. S. Varley, D. H. Nagaraju and T. Ramakrishnappa, Anal. Methods, 2014, 6, 8698-8705.

41 J. Wang, C. Bian, J. Tong, J. Sun and S. Xia, Electroanalysis, 2012, 24, 1783-1790.

42 Y. Zhang, M. Qi and G. Liu, Electroanalysis, 2015, 27, 11101118.

43 D. Wang, F. Zhang and J. Tang, Electrochemistry, 2015, 83, 84-90. 